\title{
Improved measurements of the energy and shower maximum of cosmic rays with Tunka-Rex
}

\author{
D. Kostunin*1, P.A. Bezyazeekov ${ }^{2}$, N.M. Budnev², D. Chernykh' ${ }^{2}$, O. Fedorov ${ }^{2}$
}

O.A. Gress ${ }^{2}$, A. Haungs ${ }^{1}$, R. Hiller ${ }^{1}$; T. Huege ${ }^{1}$, Y. Kazarina ${ }^{2}$, M. Kleifges ${ }^{3}$, E.E. Korosteleva ${ }^{4}$, O. Krömer ${ }^{3}$, L.A. Kuzmichev ${ }^{4}$, V. Lenok ${ }^{1}$, N. Lubsandorzhiev ${ }^{4}$, T. Marshalkina ${ }^{2}$, R.R. Mirgazov ${ }^{2}$, R. Monkhoev ${ }^{2}$, E. Osipova ${ }^{4}$, A. Pakhorukov ${ }^{2}$, L. Pankov ${ }^{2}$, V.V. Prosin ${ }^{4}$, F.G. Schröder ${ }^{1}$, A. Zagorodnikov ${ }^{2}-$ Tunka-Rex Collaboration

${ }^{1}$ Institut für Kernphysik, Karlsruhe Institute of Technology (KIT), Karlsruhe, Germany

${ }^{2}$ Applied Physics Institute of Irkutsk State University, Irkutsk, Russia

${ }^{3}$ Institut für Prozessdatenverarbeitung und Elektronik, Karlsruhe Institute of Technology (KIT), Karlsruhe, Germany

${ }^{4}$ Skobeltsyn Institute of Nuclear Physics MSU, Moscow, Russia

E-mail: dmitriy.kostunin@kit.edu

The Tunka Radio Extension (Tunka-Rex) is an array of 63 antennas located in the Tunka Valley, Siberia. It detects radio pulses in the $30-80 \mathrm{MHz}$ band produced during the air-shower development. As shown by Tunka-Rex, a sparse radio array with about $200 \mathrm{~m}$ spacing is able to reconstruct the energy and the depth of the shower maximum with satisfactory precision using simple methods based on parameters of the lateral distribution of amplitudes. The LOFAR experiment has shown that a sophisticated treatment of all individually measured amplitudes of a dense antenna array can make the precision comparable with the resolution of existing optical techniques. We develop these ideas further and present a method based on the treatment of time series of measured signals, i.e. each antenna station provides several points (trace) instead of a single one (amplitude or power). We use the measured shower axis and energy as input for CoREAS simulations: for each measured event we simulate a set of air-showers with proton, helium, nitrogen and iron as primary particle (each primary is simulated about ten times to cover fluctuations in the shower maximum due to the first interaction). Simulated radio pulses are processed with the Tunka-Rex detector response and convoluted with the measured signals. A likelihood fit determines how well the simulated event fits to the measured one. The positions of the shower maxima are defined from the distribution of chi-square values of these fits. When using this improved method instead of the standard one, firstly, the shower maximum of more events can be reconstructed, secondly, the resolution is increased. The performance of the method is demonstrated on the data acquired by the Tunka-Rex detector in 2012-2014.

35th International Cosmic Ray Conference - ICRC2017

10-20 July, 2017

Bexco, Busan, Korea

\footnotetext{
*Speaker.

${ }^{\dagger}$ now at the University of Zürich
} 


\section{Introduction}

Recent studies [1] have shown that we understand the radio emission from air-showers created by ultra-high energy ( $>100 \mathrm{PeV}$ ) cosmic rays on a level of better than $20 \%$. Theoretical calculations show, that the resolution of a radio detector operating in frequency band of $30-80 \mathrm{MHz}$ under ideal conditions can be $4 \%$ for the electromagnetic energy [2] and $11 \mathrm{~g} / \mathrm{cm}^{2}$ for the depth of shower maximum [3], Meanwhile experiments give a resolution of $15 \%$ [4] and $20 \mathrm{~g} / \mathrm{cm}^{2}$ [5], respectively, and one has to keep in mind, that the last result is obtained with small, but dense array with statistical sensitivity limit to few hundreds PeV.

Tunka-Rex, a radio detector deployed in 2012 (for details see Ref. [6, 7]), is a sparse array suited for the detection of ultra-high energy cosmic rays using the frequency band of 30-80 MHz. Up to now, for the reconstruction of air-shower parameters (primary energy and depth of shower maximum) Tunka-Rex used a method based on the LDF (lateral distribution function) [8]. By this method we performed a first direct cross-check between reconstructions provided by radio and optical (air-Cherenkov) measurements demonstrating a resolution of Tunka-Rex of $15 \%$ for the primary energy and $40 \mathrm{~g} / \mathrm{cm}^{2}$ for the depth of shower maximum. The idea of this method was based on the basic features of the radio emission: geomagnetic and Askaryan emissions, and an exponential LDF (see Refs. [9, 8] for details). Particularly, the energy is reconstructed as proportional to the amplitude at $120 \mathrm{~m}$ axis distance and the distance to the shower maximum is proportional to the slope of LDF at $180 \mathrm{~m}$ axis distance.

In the present work we develop a completely new analysis method for the Tunka-Rex experiment, which combines sophisticated signal processing using matched filtering and the statistical top-down approach developed by LOFAR [5]. Similar to LOFAR, our method requires a set of CoREAS [10] simulations to cover the range of possible shower-to-shower fluctuations.

\section{Initial dataset and simulation procedure}

Since 2015, Tunka-Rex has two different types of trigger: by dense air-Cherenkov array Tunka-133 operating during winter moonless nights, and Tunka-Grande scintillator array operating the rest of the time. As first step, only events triggered by the air-Cherenkov detector Tunka-133 in 2012-2014 are selected for this work which brings two advantages compared to Tunka-Grande triggered events. First, Tunka-133 features $6 \mathrm{~m}$ resolution for the core reconstruction [11], while the combined resolution of Tunka-Grande and Tunka-Rex is only about $20 \mathrm{~m}$ [12] since Tunka-133 is denser. Having worse resolution, the core would have to be included as free parameter, which would make computations (simulations) more complex and introduce additional systematics. Second, Tunka-133 reconstructs the depth of shower maximum and primary energy with precisions of about $28 \mathrm{~g} / \mathrm{cm}^{2}$ and $10 \%$, respectively [11] which can be used to estimate the resolution of the updated Tunka-Rex analysis by cross-comparison. Moreover, Tunka-133 reconstruction for 2015-2016 is kept in secret for the further cross-calibration with Tunka-Rex.

To obtain the initial configuration set, the events of the standard Tunka-Rex analysis [4] are taken, i.e. the simulations are configured with the shower core reconstructed by Tunka- 133 and the primary energy reconstructed by Tunka-Rex. CoREAS simulations are produced using different primary particles: protons, helium, nitrogen and iron nuclei. The exact Tunka-Rex layout rele- 
vant for each measured event is used, i.e. for the events measured in 2012-2014 the signals at a maximum of 25 antenna stations are simulated. The number of simulations per event is selected dynamically in order to cover the possible shower-to-shower fluctuations, in other words, to cover the range of possible values of shower maxima for the energy of the event. Thus, about 50 simulations per event are produced.

\section{Signal processing}

CoREAS, a simulation software for radio emission from air-showers, calculates the electricalfield vector at each Tunka-Rex antenna station. These electrical fields are convoluted with the inverted hardware response to obtain the simulated ADC counts in every channel using the Offline software [13]. After this, the measured and simulated ADC counts are convoluted with the direct hardware response of Tunka-Rex and digital filters from the standard analysis. The resulting signal traces are upsampled by a high factor $(16$ in our case) to obtain sub-ns sampling $(16 \times 200 \mathrm{MS} / \mathrm{s})$ for precise determination of the peak time.

The coordinates of reconstructed electrical fields are converted to the geomagnetic coordinate system (see Ref. [9]), and only the strongest component, namely the $\mathbf{V} \times \mathbf{B}$ component, is considered in this proceeding. Let us define the measured and simulated traces as $E_{\mathrm{m}}$ and $E_{\mathrm{s}}$, respectively.

By design, the simulated trace $E_{\mathrm{s}}$ contains no background and the peak inside it can be easily found Then the subtrace with $\pm 25 \mathrm{~ns}$ around peak is taken as signal template $v(t)$. Finding a peak in a measured trace is a more complicated problem. Due to systematic uncertainties in timing and distortion by background, the position of the measured signal is unknown, moreover the full trace can contain RFI peaks, which might be recognized as false positives. To define the signal peak and its signal-to-noise ratio (SNR) there are two windows selected in $E_{\mathrm{m}}$ similar to the standard analysis: a signal window $E_{\mathrm{m} \text {,signal }}$ and a noise window $E_{\mathrm{m}, \text { noise }}$. Thus, we apply matched filtering to $E_{\mathrm{m} \text {,signal }}$ and define the peak time as $t_{0}$. Fig. 1 shows an example of such filtering. For the further processing (fitting) we decided to consider the filtered signals $u(t)$ with a width of $t_{w}=30 \mathrm{~ns}$ around the pulse peak, since broader timeseries are much affected by background, i.e. after matched filtering the template signal $v(t)$ is also shortened to this window.

After the filtering and peak determination the following quality cuts are applied for every filtered signal $u(t)$ :

- SNR cut. We define the signal-to-noise ratio as

$$
\mathrm{SNR}=\left(\frac{\max \left(E_{\mathrm{m}, \text { signal }} * v\right)}{\operatorname{RMS}\left(E_{\mathrm{m}, \text { noise }} * v\right)}\right)^{2},
$$

and select measured signals, which have SNR $>6$ for every simulated signal.

- Peak drifting cut. This is a very strong and useful cut preventing false positives. Let us have peak times $t_{1}, t_{2}, \ldots, t_{N}$ given after matched filtering of $N$ simulated signal templates. For this set we apply the following cut: $\forall i, j \leq N: t_{i}-t_{j}<5 \mathrm{~ns}$. In other words, each simulation must predict the same peak in the trace, otherwise the peak considered as false positive and the antenna station is rejected for the complete analysis of the event. 

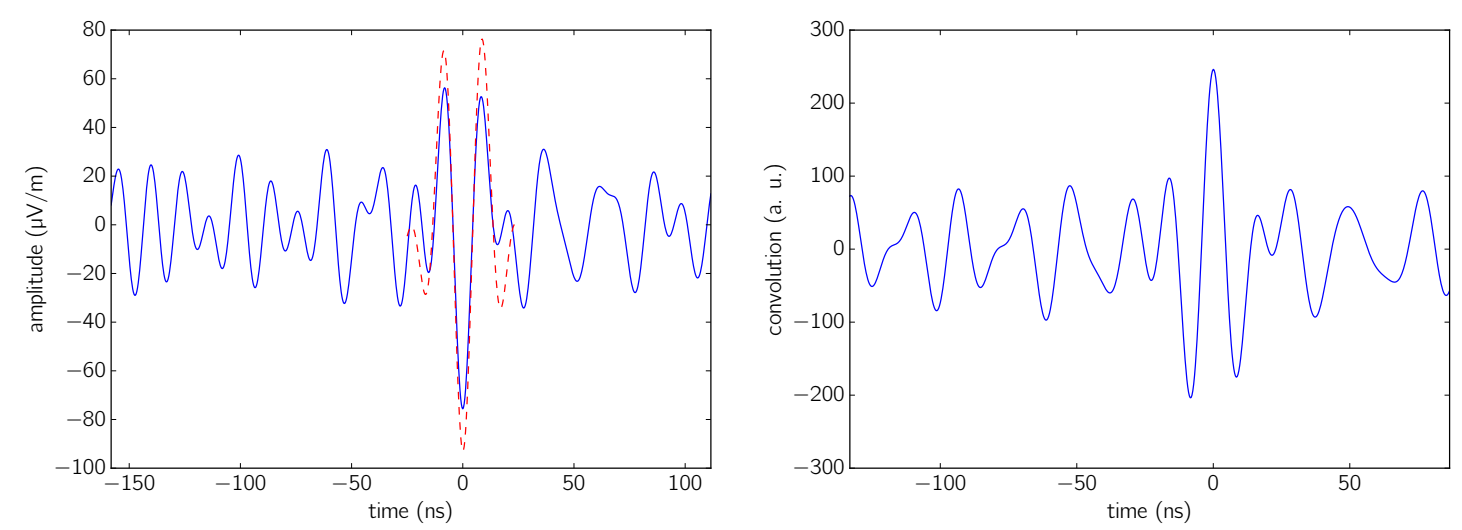

Figure 1: Left: Tunka-Rex trace $E_{\mathrm{m}, \text { signal }}$ (solid) in signal window in comparison with simulated signal $v(t)$ (dashed). Right: Convolution of measured and simulated signals. The peak of the left and maximum of the right timeseries are shifted by $t_{0}$ to be centered around zero for illustration purposes. One can note that the pulse shapes are in best agreement in $\pm 15 \mathrm{~ns}$ around peak, which is taken as window for further analysis.
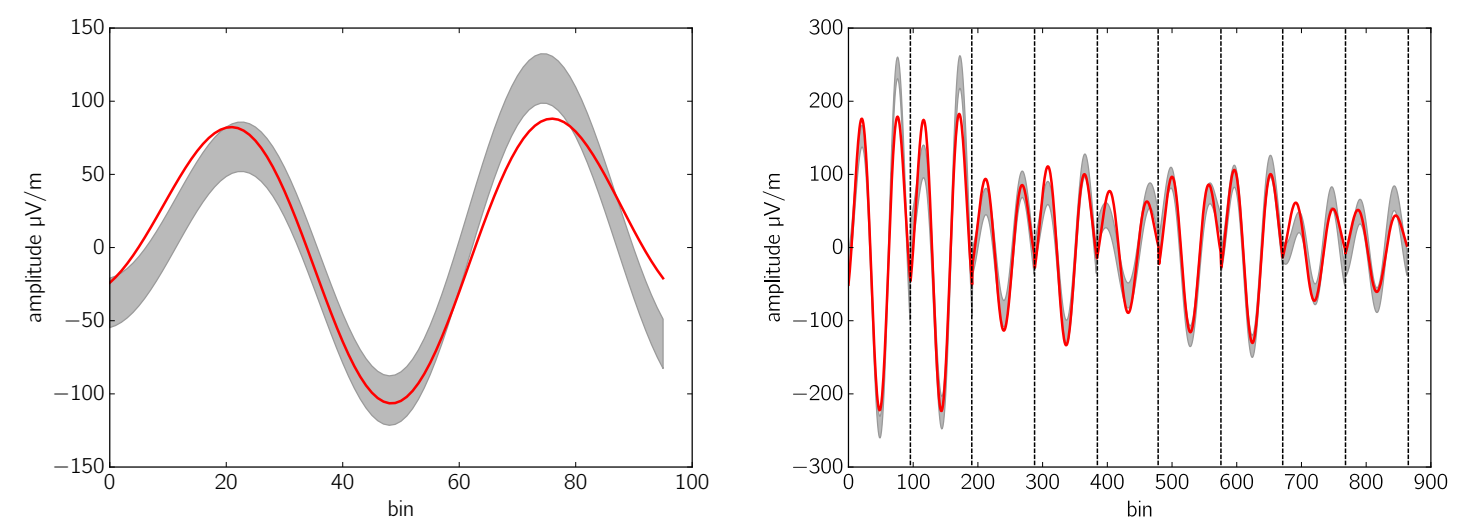

Figure 2: Example of the measured traces $u(t)$ (shaded area includes uncertainties $\sigma$ ) and the best fit $A v(t)$ (red solid line inside shaded area). Left: Example of event containing only one station with signal. Right: Example of an event with the maximum number of stations (nine). Traces are concatenated for simplicity. The vertical dashed lines are for distinction of signals from different stations.

\section{Reconstruction of air-shower parameters}

Similar to the LOFAR approach [5] for each simulation we minimize the following $\chi^{2}$ :

$$
\chi^{2}=\sum_{i=1}^{N} \sum_{t}\left(\frac{u_{i}(t)-A v_{i}(t)}{\sigma}\right)^{2}
$$

where $i$ is the index over stations with signals, the measured $u(t)$ and simulated $v(t) . t$ is the bin in the signal timeseries, $A$ is free parameter for normalization, $\sigma$ is the RMS of noise (defined in noise window $\left.E_{\mathrm{m}, \text { noise }}\right)$. Two examples of the fit is given in Fig. 2.

After the minimization of $\chi^{2}$ for each simulation, the distribution of $\chi^{2}$ over the shower maximum $X_{\max }$ for is fitted each event with a parabola, of which the minimum is the reconstructed $X_{\max }$ 

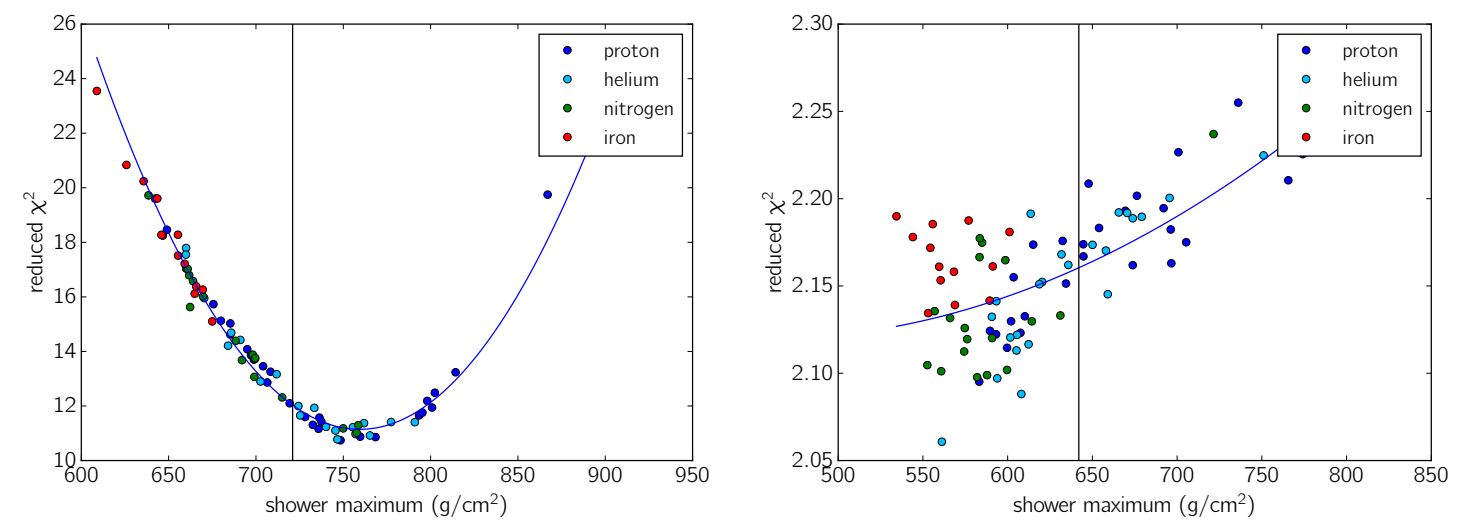

Figure 3: Example of the $X_{\max }$ distributions over reduced $\chi^{2}$. The minimum of the fitted parabola is the reconstructed $X_{\max }$, the vertical line indicates the $X_{\max }$ reconstructed by Tunka-133. On the right plot one can see, that the $\chi^{2}$ distribution is almost flat and the minimum of parabola is outside of the covered range. This indicates that the measurement of this event is not sensitive to the shower maximum, and the event is rejected.

(see Fig. 3). We omit events, which have a reconstructed $X_{\max }$ outside of the of simulated range (see Fig. 3, right). After this, we obtained twice more events with shower maximum reconstruction compared to the old Tunka-Rex analyses [4]. Cross-check with the Tunka-133 reconstruction have shown that both experiments are in agreement in absolute scales, and the resolution of the $X_{\max }$ reconstruction with the new method is about $35 \mathrm{~g} / \mathrm{cm}^{2}$. The comparison between values reconstructed by Tunka-133 and Tunka-Rex is given in Fig. 4.

The energy reconstruction was not the main focus of the present work, by this the preliminary reconstruction of the primary energy is performed with simple method of taking initial energy normalized by $A$ with minimal $\chi^{2}$ for events left after fitting. Since the correction for the mass composition is in progress, this normalization is performed for each primary particle separately. The results of the energy reconstruction in comparison with Tunka-133 is given in Fig. 5. Comparing to the old reconstruction we obtained systematic shift of about $10 \%$, which can be explained by the distortion introduced by noise, which can contribute up to $25 \%$ of amplitude for small SNRs. Since radio is already used for the absolute scale comparison between different cosmic-ray experiments [14], the accurate estimation of the primary energy is of special importance for the radio.

Although the development of the new method discussed in the present work is in progress, there are significant improvement of the reconstruction comparing to the old Tunka-Rex analysis. The summary of performance of both methods ${ }^{1}$ is given in Table 1 .

\section{Conclusion and discussion}

We made a further step in the improvement of the shower maximum reconstruction with sparse radio arrays, which will result in a mass composition study in the energy range of $10^{17}-10^{18} \mathrm{eV}$. With the exposure accumulated in 2012-2017 [15], Tunka-Rex will be able to study the $\mathrm{X}_{\max }$ dis-

\footnotetext{
${ }^{1}$ In the present analysis the phase correction mentioned in Ref. [7] is not implemented.
} 

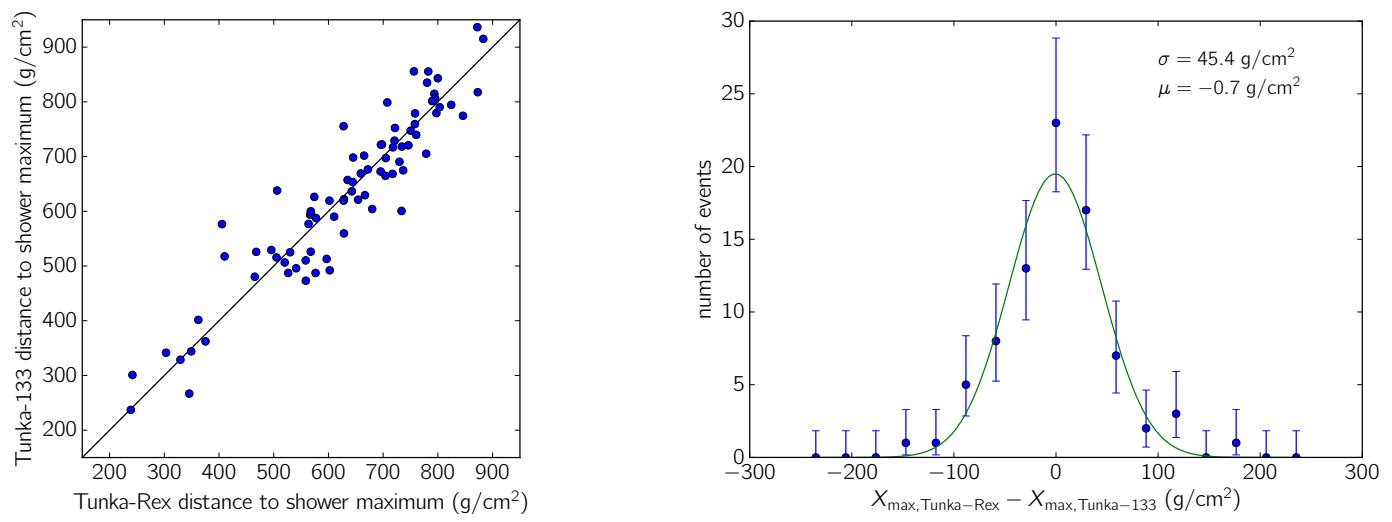

Figure 4: Correlation and difference of the distance from the detector to $X_{\max }$ as reconstructed with TunkaRex radio and Tunka-133 air-Cherenkov measurements.
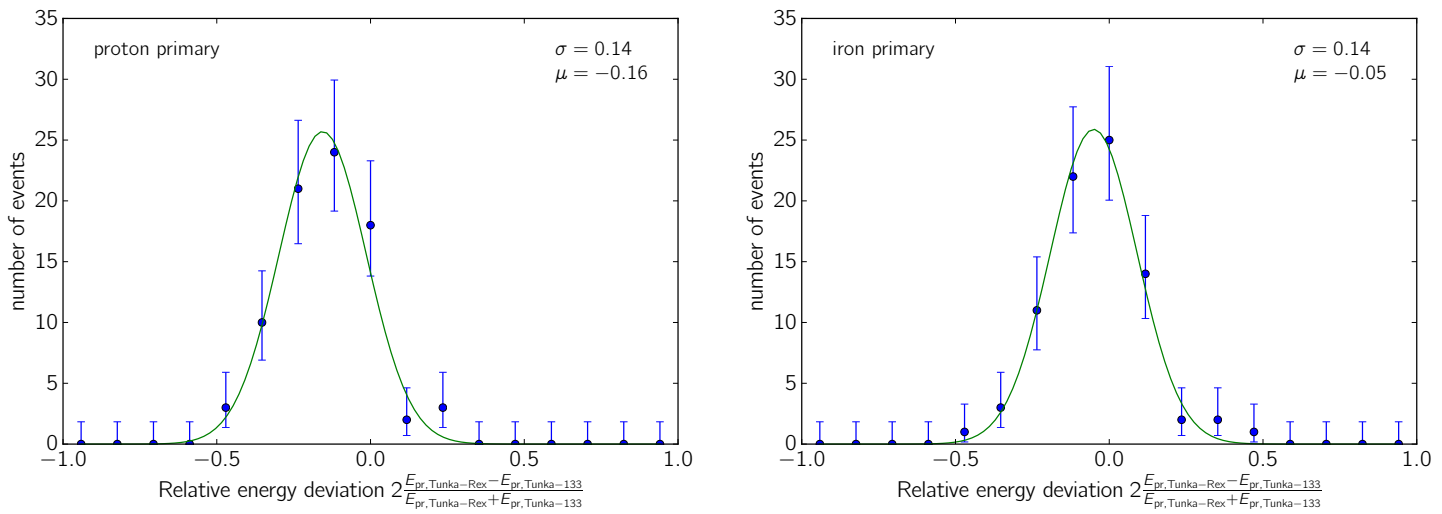

Figure 5: Relative deviation of the shower energy reconstructed with the Tunka-133 air-Cherenkov and the Tunka-Rex radio measurements in assumption of proton (left) and iron (right) primaries. One can see, that the iron assumption gives larger primary energy due to a smaller electromagnetic energy deposit.

Table 1: Comparison chart of the performance between the old and new Tunka-Rex analyses. Resolutions for $E_{\mathrm{pr}}$ and $X_{\max }$ are derived after substraction of the Tunka-133 values of $10 \%$ and $28 \mathrm{~g} / \mathrm{cm}^{2}$, respectively.

\begin{tabular}{lll} 
Property & Old method & New method \\
\hline Number of events passing quality cuts (of 183) & 44 & 81 \\
$E_{\text {pr }}$ resolution & $10 \%$ & $10 \%$ \\
$X_{\max }$ resolution & $40 \mathrm{~g} / \mathrm{cm}^{2}$ & $35 \mathrm{~g} / \mathrm{cm}^{2}$
\end{tabular}

tribution for these energies with statistics comparable to ones already collected by existing optical detectors.

The results presented in this proceeding are still preliminary and there are important changes, which we plan to implement soon:

- More accurate estimation of uncertainties and biases introduced by noise. For the time being, as signal uncertainty we use the RMS in the noise window, however this uncertainty can 
be estimated more precisely, which will improve the quality of the $\chi^{2}$ fit. We will also study whether the bias of about $10 \%$ in energy reconstruction compared to the standard reconstruction is due to noise.

- Stronger quality cuts have to be defined. In the present work we do not introduce any postreconstruction quality cuts, however with increased statistics such quality cuts can be used to select events reconstructed with higher accuracy.

Generally, there is space for the further improvements of the method. Simulations shows, that the resolution of the detector under realistic conditions can be at least $25 \mathrm{~g} / \mathrm{cm}^{2}$ with noise and $15 \mathrm{~g} / \mathrm{cm}^{2}$ without noise. It is possible, that whitening the noise, e.g. with a median filter, and more accurate tuning of the matched filtering and quality cuts could improve the reconstruction. On the other hand, there are more complex uncertainties, e.g. by atmosphere conditions [16].

One of the main advantages of this method is the reconstruction the air-shower parameters exploiting the pulse shape informationa and not only one point per antenna stations (amplitude or power). Using the shower geometry of the triggering detector, not only the energy can be reconstructed from a single antenna station [17], but also $X_{\max }$, which lowers the threshold and increases the aperture of radio extensions to particle detector arrays.

\section{Acknowledgements}

The construction of Tunka-Rex was funded by the German Helmholtz association and the Russian Foundation for Basic Research (grant HRJRG-303). This work has been supported by the Helmholtz Alliance for Astroparticle Physics (HAP), by Deutsche Forschungsgemeinschaft (DFG grant SCHR 1480/1-1), by the Russian Federation Ministry of Education and Science (14.B25.31.0010, 2017-14-595-0001-003, No3.9678.2017/8.9, No3.904.2017/4.6, 3.6787.2017/7.8, 1.6790.2017/7.8), by the Russian Foundation for Basic Research (grants 16-02-00738, 16-32-00329, 17-02-00905), and by grant 15-12-20022 of the Russian Science Foundation (section 4).

\section{References}

[1] F. G. Schröder, Radio detection of Cosmic-Ray Air Showers and High-Energy Neutrinos, Prog. Part. Nucl. Phys. 93 (2017) 1-68, [1607.08781].

[2] C. Glaser et al., Simulation of the Radiation Energy Release in Air Showers, EPJ Web Conf. 135 (2017) 01016, [1609.05743].

[3] A. Zilles, S. Buitink and T. Huege, Initial simulation study on high-precision radio measurements of the depth of shower maximum with SKAI-low, EPJ Web Conf. 135 (2017) 02004, [1702. 0 0283].

[4] P.A. Bezyazeekov, et al. - Tunka-Rex Coll., Radio measurements of the energy and depth of maximum of cosmic-ray air showers by Tunka-Rex, JCAP 01 (2016) 052.

[5] S. Buitink et al., Method for high precision reconstruction of air shower $X_{\max }$ using two-dimensional radio intensity profiles, Phys. Rev. D90 (2014) 082003.

[6] TUNKA-ReX collaboration, P. A. Bezyazeekov et al., Measurement of cosmic-ray air showers with the Tunka Radio Extension (Tunka-Rex), Nucl. Instrum. Meth. A802 (2015) 89-96, [1509.08624]. 
[7] F.G.Schröder et al. (Tunka-Rex Collaboration), Overview on the Tunka-Rex antenna array for cosmic-ray air showers, in Proceedings of the 35th International Cosmic Ray Conference 2017, Busan, Korea, p. PoS (ICRC2017) 459, 2017.

[8] D. Kostunin et al., Reconstruction of air-shower parameters for large-scale radio detectors using the lateral distribution, Astropart. Phys. 74 (2016) 79.

[9] TUNKA-REX collaboration, D. Kostunin et al., Towards a cosmic-ray mass-composition study at Tunka Radio Extension, EPJ Web Conf. 135 (2017) 01005, [1611.09127].

[10] T. Huege, M. Ludwig and C. James, Simulating radio emission from air showers with CoREAS, AIP Conf.Proc. 1535 (2013) 128, [1301.2132].

[11] V. V. Prosin et al., Results and perspectives of cosmic ray mass composition studies with EAS arrays in the Tunka Valley, J. Phys. Conf. Ser. 718 (2016) 052031.

[12] D. Kostunin et al., Latest results of the Tunka Radio Extension, EPJ Web Conf. 145 (2017) 11001, [1701.07165].

[13] Pierre Auger collaboration, P. Abreu et al., Advanced functionality for radio analysis in the Offline software framework of the Pierre Auger Observatory, Nucl.Instrum.Meth. A635 (2011) 92-102, [1101.4473].

[14] TUNKA-REX, LOPES collaboration, W. D. Apel et al., A comparison of the cosmic-ray energy scales of Tunka-133 and KASCADE-Grande via their radio extensions Tunka-Rex and LOPES, Phys. Lett. B763 (2016) 179-185.

[15] O. Fedorov et al. (Tunka-Rex Collaboration), Detector efficiency and exposure of Tunka-Rex for cosmic-ray air showers, in Proceedings of the 35th International Cosmic Ray Conference 2017, Busan, Korea, p. PoS (ICRC2017) 387, 2017.

[16] A. Corstanje et al., The effect of the atmospheric refractive index on the radio signal of extensive air showers, Astropart. Phys. 89 (2017) 23-29, [1701.07338].

[17] TUNKA-REX collaboration, R. Hiller et al., Tunka-Rex: energy reconstruction with a single antenna station, EPJ Web Conf. 135 (2017) 01004, [1611.09614]. 